\title{
The Use of Finite Differences on Electric Currents Gives Credit to Coulomb's Law as Causing Electromagnetic Forces, thereby Explaining Electromagnetic Induction
}

\author{
Jan Olof Jonson
}

\begin{abstract}
Using differential calculus rigorously on electric currents, thereby using the method of finite elements, it has been possible to derive expressions for the total electromagnetic force between two electric currents, based solely on Coulomb's law, thereby applying the effects of propagation delay. These results, explored in earlier publications, have been used in order to attain a new explanation to electromagnetic induction, thereby simultaneously rejecting Faraday's Law of Induction. It is being shown that Faraday's Law of Induction fails to account for the behavior of the induced signal in a secondary loop of a typical transformer circuit, since it has been found a mathematical deficiency in the traditional analysis of electric measurements, which implies a phase fault. Instead it is successfully being shown that the usage of the Displacement Current Density, based on Coulomb's Law, and the Continuity Equation of Electricity are able to account for the correct phase behavior.
\end{abstract}

Index Terms-Coulomb's law, Faraday's law of induction, the displacement current density, the continuity equation of electricity, new definition of propagation delay, transformer circuit, wrong analysis of measurements

\section{INTRODUCTION}

In an earlier publication it has successfully been shown that the electromagnetic force between two current carrying conductors can satisfactorily be explained using Coulomb's original force law of 1785 [1]

It was not needed to use the Lorentz Force. Furthermore, the Lorentz force even fails to predict the qualitative behavior of the force within Ampere's Bridge, as measured by Pappas and Moyssides in the early 1980's [2]. The Lorentz force gives no dependence of the wire thickness, whereas Coulomb's law does, thereby being in accordance with measurements [3].

It was in this connection not necessary to involve any magnetic fields, contrary to common practice. The new theory is unique in that it is based on Coulomb's law and it therefore implies a simpler explanation to induction. A consequence of the discovery that it is the electrostatic field according to Coulomb's law that is responsible for the electromagnetic force also between electric currents, is that electrostatics may also be used in order to explain electromagnetic induction. Of course it does not sound very

Manuscript received May 8, 2013; revised July 2, 2013.

Jan Olof Jonson is with the Stockholm University, SE-10691 Stockholm, Sweden (e-mail: universitetet@su.se). strictly to speak of 'electrostatics' in cases, where charges are moving, but it is the originally electrostatic laws, which appear to be usable also in the electrodynamics cases, without involving any additional use of magnetic fields. Coulombs' law combined with the 'Continuity Equation of Electricity' can give credit to the induced current in the secondary circuit of a transformer circuit.

\section{How COULOMB's LAW CAN BE USED IN} EXPLAINING THE FORCE BETWEEN ELECTRIC CURRENTS

In a paper 1997 [1] the electromagnetic force between two current carrying conductors has been derived using solely Coulomb's law, instead of using Biot-Savart's law and the Lorentz force law. Coulomb's law is applied directly on the point charges, which constitute the respective current, thereby taking into account the effects of the different propagation delay between different parts of the respective conductors [3]. Contrary to the customary description of the effects of propagation delay and retarded action as explored by Jackson [4], propagation delay will appear both with respect to the sending point and the target point of the electric force vector. When the propagation delay is derived in that way, the force between electric currents, usually ascribed the Lorentz force law, can be derived, solely using Coulomb's law. Jackson is exploring the current view [4], saying that (thereby treating the usage of the LiénardWiechert potentials) that the "quantity in the brackets is to be evaluated at the retarded time". This approach is repeated, when deriving the electric field as well [5]. To conclude, the customary method only takes into account the retardation effects with respect to the starting point of propagation, thereby completely missing the effects when a propagated field arrives at the current with which it is reacting. Hence, he is talking only about retardation with respect to the point at a current, from which action is being propagated. Furthermore, that the new interpretation of Coulomb's law described above [3] is usable, is shown by other experiments. It has for example been possible to derive the electromagnetic force between the two parts of a set of Ampère's Bridge correctly, whereas the Lorentz force law fails. So there is strong experimental evidence supporting this new interpretation of Coulomb's law. This is the basis for applying Coulomb's law also on the case of electromagnetic induction, which will be done in the following. A theory that fails in one case must be abandoned and replaced by a new theory, being able to explain the measurements. 


\section{DERIVING AN EXPRESSION FOR ELECTROMAGNETIC INDUCTION}

\section{A. The Analysis of a Primary Circuit}

It will be assumed a simple transformer circuit, with a voltage generator with negligible inner impedance, a primary winding and a volt meter attached parallel to the voltage generator with attached primary winding. For simplicity, no iron or ferrite core has been applied. This gives rise to the following figure.

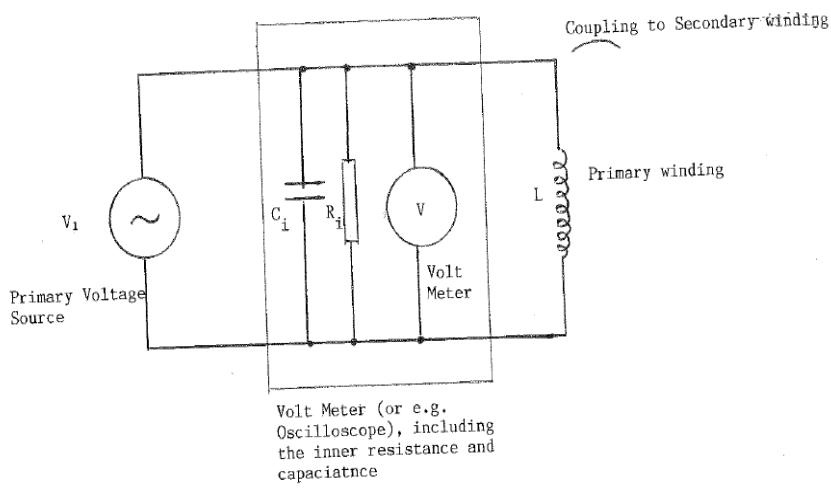

Fig. 1. The primary side of a transformer circuit

Since it has been claimed [6] that a volt meter reacts on the current that goes through it, it is therefore of interest to now define that current, giving it the name $I_{v 1}$. Using the standard formalism within electric circuit theory, referring to sine signals, one will attain

$$
I_{v 1}=\frac{V_{1}}{\frac{1}{i \omega L}+\frac{1}{R_{i}}+i \omega C_{i}} \cdot \frac{i \omega L}{i \omega L+\frac{R_{i} \cdot \frac{1}{i \omega C_{i}}}{R_{i}+\frac{1}{i \omega C_{i}}}}
$$

Analyzing the behavior of this filter function with respect to the applied frequency, it is reasonable to assume that the frequency is well below the breakdown level with respect to the inner capacitance of the volt meter/oscilloscope. This assumption leads to the approximation valid for middle range frequencies up to the border frequency being of order $1 / R_{i} C_{i}$.

$$
I_{v 1} \cong \frac{V_{1}}{R_{i}}
$$

Too low frequencies have to be avoided due to the shortcut effect of the inductor in that case. In a more formal way one would speak of a 'band pass filter function'.

During these circumstances there will be no phase difference between current and voltage with respect to the volt meter.

\section{B. Implications for the Secondary Circuit}

The secondary circuit of a simple transformer circuit has been analyzed elsewhere [6]. Due to the discoveries concerning the usability of the relationship between the
Electric Displacement and the induced current described in that paper, a current will be induced in any conductor which is affected by a varying electric displacement, independently of where this field has been generated. This statement makes it possible to abandon the idea of 'magnetic fields', whose time variation is supposed to give rise to an induced emf (electromotive force) in a secondary winding, according to today's widely recognized theory.

Accordingly, in the secondary winding, a current $I_{2}$ is induced according to the law of the Electric Displacement, applied to the actual dimensions of a conducting wire:

$$
I_{2}=-\frac{\varepsilon_{0} \varepsilon_{r} A}{L} \cdot \frac{\partial V_{2}}{\partial t}
$$

The law of the Electric Displacement originally reads

$$
\vec{i}=-\frac{\partial \vec{D}}{\partial t}
$$

thereby neglecting the constant term that appears in the right hand term in the traditional interpretations. In this simplistic case no external field sources, constant in time are assumed to exist.

To be noted is that the common usage of electric displacement has been in capacitors, or more generally in non-conducting media.

$V_{2}$ is the potential drop along the secondary winding. If the primary and secondary windings are close to each other, and of equal length, on may simply write

$$
V_{2}=k \cdot V_{1}
$$

valid as a good first order approximation, which is sufficient for this analysis.

Hence, it will not be any substantial phase shift between the primary and the secondary winding.

In the vicinity of the primary winding there will namely not appear any substantial potential loss compared to the inner of itself. For practical reasons the analysis is neglected.

where $k$ is rather close to one,

$$
k \cong 1
$$

It has been shown elsewhere [7] that the voltage $V_{v}$ of the volt meter applied to the secondary circuit will show a signal lagging 90 degrees behind the voltage $V_{2}$, induced in the secondary winding. The figure describing this scenario is repeated here [8].

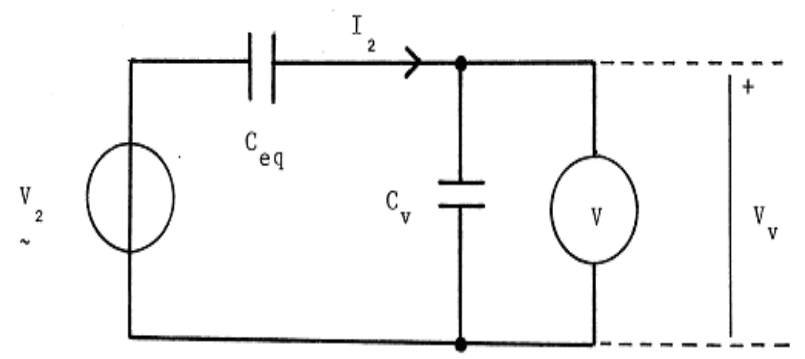

Fig. 2. Schematic of the secondary circuit of a typical, simplistic transformer circuit [6] 
Since $V_{2}$ will be approximately in phase with $V_{1}$, as a consequence of Eq. (5) above, and the displayed voltage in the latter case will be proportional to the current $I_{1}$ that is going through it, as a consequence of Eq. (2) above, the displayed voltage $V_{v}$ of the secondary circuit will lag $90^{\circ}$ behind the displayed voltage $V_{1}$ of the primary circuit.

Hence, the total phase shift from the original voltage to the output voltage, as recorded by volt meters or oscilloscopes will be $-90^{\circ}$.

In the same paper it was also shown that the phase shift that would occur, provided the Induction Law was to be valid, would be $-180^{\circ}$ [9], which does not fit with reality.

\section{Other Critics of Faraday's Law of Induction}

Wells [10], [11] is presenting several critical points against Faraday's law of induction. He claims that Faraday's law and especially its expression through Maxwell's fourth equation, directly violate both conservation laws, the First Law of Thermodynamics and the Third Law of Motion.

Kholmetskii [12] is critical against Faraday's law of induction, claiming that there will appear internal electromagnetic fields within a conducting circuit which implies a violation of Faraday's law.

Macleod [13] discusses the related phenomenon of unipolar induction, referring to a series of experiments showing that, contrary to common view, that the source of the emf generated by rotation of a conductive disk, having a cylindrically symmetrical magnet attached coaxially to it, is situated in the disk, not in the external galvanometer circuit.

Heras [14] discusses the true nature of the displacement current in connection with Faraday's induction. This is especially interesting, since the actual conference paper makes use of the displacement current in order to explain electromagnetic induction.

\section{CONCLUSIONS}

Two fundamental discoveries have been further explored here; firstly that a time-varying electric field gives rise to an induced current in a secondary winding provided it is a part of a closed; secondly that the secondary circuit can be described mainly in terms of capacitances. It was possible to derive the secondary current in the described way if using earlier results concerning the more general applicability of Coulomb's Law, thereby rejecting the very existence of separate 'magnetic fields'. The application of a capacitance in order to describe the generation of the electric current in the secondary winding was a consequence of the usage of the Law of electric Displacement. This in turn implied an additional 90 degrees phase shift compared to the commonplace model of a transformer circuit, and, hence the Induction Law brings about a 90 degrees to large phase shift, but the recently discovered model is able to account for the usual 90 degrees phase shift.

The deeper consequence of these combined results described above is that the commonplace model of today must be rejected and that new corroborative arguments have been set forth favoring the new Coulomb based model.

\section{UNITS}

$V_{1}$ Voltage of the primary voltage source

$C_{i}$ The inner capacitance of a volt meter attached to the primary voltage source

$R_{i}$ The inner resistance of a volt meter attached to the primary voltage source

$\checkmark$ Volt meter

$L$ The inductance of the primary winding

$I_{v 1}$ The current that goes through the volt meter of the primary circuit

$\omega$ Angular frequency of the signal of the primary voltage source

$I_{2}$ The current that is induced in the secondary winding

$\varepsilon_{0}$ Vacuum permittivity

$\varepsilon_{r}$ Relative permittivity

$A$ Area of the conducting the secondary winding

$V_{2}$ The electric potential drop along the secondary winding

$k$ Numerical constant defining the proportionality between $V_{1}$ and $V_{2}$

$t$ Time

$\vec{i}$ Current density in the secondary winding

$\vec{D}$ Electric displacement field inside the secondary winding

$C_{e q}$ The equivalent capacitance of the total secondary circuit, including both the secondary winding and the volt meter or oscilloscope

$C_{v}$ The equivalent capacitance of the attached volt meter or oscilloscope

$V_{v}$ Registered voltage of an applied volt meter or oscilloscope, connected to the secondary winding

\section{REFERENCES}

[1] J. O. Jonson, "The Magnetic Force between Two Currents Explained Using Only Coulomb's Law," Chinese Journal of Physics, vol. 35 no. 2, pp. 139-149, April 1997.

[2] P. G. Moyssides and P. T. Pappas, "Rigorous quantitative test of Biot-Savart-Lorentz forces," Journal of Applied Physics, vol. 59, pp.19-27, issue 1, 1986.

[3] J. O. Jonson, "The Magnetic Force between Two Currents Explained Using Only Coulomb's Law," Chinese Journal of Physics, vol. 35 no.2, pp.140, April 1997.

[4] J. D. Jackson, Classical Electrodynamics, 2nd ed. John Wiley \& Sons, 1975, Eq. (14.8), pp. 656.

[5] J. D. Jackson, Classical Electrodynamics, 2nd ed. John Wiley \& Sons, 1975, Eq. (14.8), pp. 657

[6] J. O. Jonson, "The Law of Electromagnetic Induction Proved to Be False Using Classical Electrostatics," Journal of Theoretics, vol. 5-3, Section 4, 2003

[7] J. O. Jonson, "The Law of Electromagnetic Induction Proved to Be False Using Classical Electrostatics," Journal of Theoretics, vol. 5-3, Eq. (17)

[8] J. O. Jonson, "The Law of Electromagnetic Induction Proved to Be False Using Classical Electrostatics," Journal of Theoretics, vol. 5-3, Section 4.1, Fig. 2

[9] J. O. Jonson, "The Law of Electromagnetic Induction Proved to Be False Using Classical Electrostatics," Journal of Theoretics, vol. 5-3, Section 4.2 .

[10] S. I. Wells, "Dual Dilemma from Faraday's Law? Constructive Fraud at the Foundation of Electrodynamics," in Proc. 15th Natural Philosophy Alliance Conference, Albuquerque, NM, United States, 2008. 
[11] S. I. Wells, "Faraday Pressure and Energy Conservation," in Proc. $16^{\text {th }}$ Natural Philosophy Alliance Conference, Storrs, CT, United States, 2009 .

[12] A. L. Kholmetskii, "The Faraday Induction Law and Field Transformations in Special Relativity," Apeiron, vol. 10, no. 2, 2003.

[13] N. Macleod, "Faraday's disk revisited: Some new experiments concerning unipolar electromagnetic induction," Physics Essays. Dec. 2012, vol. 25, issue 4, pp. 524-531

[14] J. A. Heras, "A Formal Interpretation of the Displacement Current and the Instantaneous Formulation of Maxwell's equations," American Journal of Physics, vol. 79, issue 4, pp. 409, April 2011.

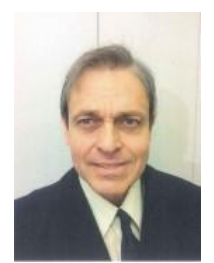

Jan Olof Jonson was born in the Town Solna of Sweden, Europe, 1956. He got his MSEE at the KTH technical university (the Royal Institute of Technology), Department of Microwave Techniques, in Stockholm, Sweden, 1982. He pursues his BSc with a Major in Meteorology at the Stockholm University, Department of Meteorology, in Stockholm, Sweden, 2012.

$\mathrm{He}$ has earned his living mainly within university education after having achieved his first academic degree in 1982, but he has also been engaged in private enterprise the last decade, all positions mainly of a short-time nature, except for a three-year position as a research engineer at the KTH technical university 1997-2000. The current jobs are a private enterprise position as a Customer Booker at the heat pump company 'Värmepumpsgruppen Åkersberga AB' at the address Båthamnsvägen 6, SE-184 40 Åkersberga, Sweden and temporary jobs as Invigilator (Proctor) at the KTH Technical University, SE-100 44 Stockholm, Sweden.

Jan Olof Jonson has published several papers mainly on Electromagnetism, beginning with a paper published by the Chinese Journal of Physics in 1997, entitled "The Magnetic Force Between Two Currents Explained Using Only Coulomb's Law", in which it is proven that Coulomb's law is able to account for the repulsive forces within Ampere's bridge, whereas the Lorentz force law is not. The latest paper was published in 2012 by the Open Access publishing company InTech, entitled "Ampère's Law Proved Not to Be Compatible with Grassmann's Force Law". A paper presenting a new photon model is published in the Proc. IX International Scientific Conf., "Towards a Classical Explanation to the Stable Electron Paths around Nuclei and to Radiation in Connection with the De-Excitation of Excited Electrons", "Space, Time, Gravitation", SaintPetersburg, Russia, 2004. 